\title{
Relation of Accelerometer and EMG Recordings for the Measurement of Upper Extremity Movement
}

\author{
Andreas Keil ${ }^{1}$, Thomas Elbert ${ }^{1}$, and Edward Taub² \\ 'Department of Psychology, University of Konstanz, Germany \\ ${ }^{2}$ Department of Psychology, University of Alabama at Birmingham, USA
}

Keywords: Accelerometry, electromyography, ambulatory monitoring, activities of daily life, standardized movements

\begin{abstract}
In order to determine the value of accelerometry as a measure of real world outcome when a subject is outside the laboratory, accelerometer recordings from the wrist were compared with simultaneous electromyogram (EMG) recordings from the lower and upper arm. Accelerometer and EMG signals were recorded simultaneously by the "Kölner Vitaport System," an ambulatory device. Six male subjects performed standardized tasks as well as activities of daily life (ADL). Low correlations between accelerometer counts and integrated EMG were found in the standardized tasks, whereas there were considerably higher correlations for ADL. However, there was a strong relation between several parameters derived from EMG and accelerometer recordings. The two techniques appear to measure different aspects of movement and may be complementary.
\end{abstract}

\section{Introduction}

Obtaining information concerning an individual's motor activity is useful for a number of both clinical and research issues as, for instance, neurological assessment (Boyce et al., 1992; Formisano, Pratesi, Modarelli, Bonifati, \& Meco 1992), developmental studies (Salminen, Oksanen, Maki, Pentti, \& Kujala, 1993), and ambulatory monitoring (Fahrenberg, Förster, Smeja, \& Müller, 1997). In particular, there is a growing recognition of an urgent need for new and ecologically more valid measures of movement in the field of physical rehabilitation (e. g., Duncan, 1997). The emphasis is on the development of "real world outcome measures," of which there are at present only a limited number and these have seri- ous limitations. This need is made more salient by the fact that there is often a very large gap between the movements that a stroke patient can perform with the affected arm when asked to carry out tasks in a laboratory motor test and what s/he will actually do with the extremity when out of the laboratory. In many cases, use in the life situation is often very much less than they show themselves capable of in a laboratory motor test, and in some cases it is virtually zero. This striking phenomenon was first reported by Andrews and Stewart (1979) and was later confirmed in another laboratory (Taub et al., 1993; Taub, Pidikiti, DeLuca, \& Crago, 1996; Taub \& Wolf, 1997). A neurorehabilitation technique has recently been developed for greatly increasing the actual amount of use of an affected extremity of chronic stroke patients in the real world, non-clinic situation (Taub, 1980; Taub et al., 
1993, 1996; Taub \& Wolf, 1997; Wolf, LeCraw, Barton, \& Jann 1989). This may be viewed as the ultimate objective of physical rehabilitation, but initially no methods were available for quantifying this therapeutic change. Self-report instruments were employed (e. g., Motor Activity Log, Taub et al., 1993), but this type of measure has several shortcomings, even when verified by the independent report of significant others. Subjective measures are prone to be influenced by effects of demand characteristics, social desirability, or misperception. Moreover, direct observation techniques in the home are costly, hard to realize, and also subject to demand characteristics. Therefore, objective methods to measure aspects of motor behavior by means of ambulatory devices as have been employed by Fahrenberg and coworkers (1996) in another context would constitute an important contribution to this type of physical rehabilitation study.

Several authors have proposed the use of accelerometers and address the question of their reliability and validity (Bouten, Verboeket van de Venne, Westerterp, Verduin, \& Janssen, 1996; Janz, 1994; Meijer, Westerterp, van Hulsel, \& ten Hoor, 1991; Richardson, Leon, Jacobs, Ainsworth, \& Serfass, 1995). To date, use of accelerometers as a valid and reliable measure of overall physical activity has been realized in a variety of conditions, such as eating disorders (Rutter, 1994), sleep disorders (Tobler, 1993), mental retardation (Repp, Karsh, Deitz, \& Singh, 1992), and cerebral palsy (Bach, Reddihough, Burgess, \& Johnson, 1994). There have also been attempts to quantify tremor (Comby, Chevalier, \& Bouchoucha, 1992) and chronocardiac variables (Otsuka, Cornelissen, \& Halberg, 1991) by means of accelerometers. In these studies, information was obtained on the subjects' locomotion or global physical activity. In contrast, for motor rehabilitation outcome studies, information is required on the specific kind and amount of movements performed with different extremities.

In the present study, we simultaneously recorded accelerometer and EMG activity in order to determine their comparability with respect to self-induced upper extremity movement. Uniaxial accelerometers were used in this study for two reasons. First, the expense of triaxial devices would probably reduce the extent to which they would be used in the field of physical rehabilitation. Second, Redmond and Hegge (1985) have shown that, while the correlation of output for the two instruments can be low during an epoch of $1 \mathrm{~min}$, with longer time intervals the correlation becomes rapidly greater, and at $4 \mathrm{~min}$ the correlation is nearly 1 . The purpose of developing accelerometry in the present context is for long-term ambulatory monitoring, where recording time epochs can be 4 min or longer. Thus, uniaxial accelerometers should be as useful as triaxial devices.

EMG was used because it is a well-established mea- sure of motor performance which has been used for decades and has been shown to be a valid and reliable indicator of muscular activity (Schandry, 1981). However, there are some problems associated with the use of EMG as a measure of movement for several reasons. Thus, isometric muscle contraction results in EMG readings without the occurrence of overt movement. Moreover, only a limited number of channels and a few muscle groups (typically $<3$ ) can be scanned. In addition, when using a single EMG channel, only movements made in a certain direction can usually be monitored. Thus, the correspondence between surface EMG recordings and actual movements that can be observed by an investigator remains generally unsatisfactory. Accelerometer data have problems as well. For example, accelerometers are sensitive to limb movements induced by gravity or by locomotion of the whole body which do not require directed actions of a limb. Therefore, one might not expect high correlations between EMG and accelerometer time series measurements for a given set of tasks. There are some occasions on which both EMG and accelerometers should respond to the same motor event. However, the correlation may be weak. It seems possible that the two measures may index different parameters of movement, especially for some movements, and that therefore they may be complementary methods for assessing the amount of extremity activity when a subject is out of the laboratory. This research is part of a more general effort to develop real world outcome measures for the field of rehabilitation (Uswatte \& Taub, in press).

\section{Methods}

\section{Subjects}

Six right-handed male subjects, who were normal with respect to motor ability, voluntarily participated in the study. Their age ranged from 22 to 49 years, with a mean of 29.3 years $(S D=9.2)$. All subjects were paid for participation.

\section{Apparatus and Materials}

Recordings were carried out by means of the "Kölner Vitaport System," an ambulatory device which is designed to have 16 bit resolution. For accelerometer recordings, two sensors with piezoelectric crystal technology were placed at the mid-dorsum of the wrist and at the lateral aspect of the hip. Both accelerometers were single-axial sensors (Vitaport GmbH, Erftstadt, Germany). The sensor placed at the hip was considered to be 
sensitive to locomotion of the subject. Since translation of the body through space would also passively carry along the arms, it was felt that this type of nonactive movement of the arms should be eliminated from the record. This was to be accomplished by subtracting hip accelerometer counts from arm accelerometer counts. EMG was recorded from the dorsal aspect of the lower arm and the ventral aspect of the upper arm (biceps EMG) using ARBO $^{\mathrm{TM}}$ self-adhesive electrodes with a contact area of $0.8 \mathrm{~cm}^{2}$ on standard recording sites as proposed, for example, by Schandry (1981). Preparation paste was used to abrade the skin at these sites in order to reduce skin resistance. In the EMG channels, data were integrated and then low-pass filtered at $12.5 \mathrm{~Hz}$. Integrated EMG was digitized at a rate of $50 \mathrm{~Hz}$. A moving average filter with a cut-off at $6.25 \mathrm{~Hz}$ was used in the accelerometer channels where the sampling rate was $200 \mathrm{~Hz}$. For the standardized tasks section of the procedure, a separate channel was employed to mark data recorded when subjects initiated task movements in order to exclude signals recorded while the subjects were listening to the instructions.

\section{Procedure}

After giving informed consent, subjects started the experimental session by completing a series of standardized tasks in the laboratory. A metronome operating at a frequency of 40 beats per minute was used as a pacer for the movements, 9 of which were taken from the Wolf Motor Function Test (see Taub et al., 1993; Wolf et al., 1989). The test movements employed were as follows: (1) raise the hand in a forward direction from lap onto table, (2) raise hand from table onto box $30 \mathrm{~cm}$ high, (3) extend and flex lower arm while sitting sideways to the table, (4) lift a can from table to mouth, (5) lift a pencil off table surface, (6) flip 3 playing cards on table from side to side, (7) stack 3 checkers on a table in front of subject, (8) fold a towel in half lengthwise in the air and then again lengthwise while towel is on the table, followed by the unfolding of the towel in preparation for the next folding repetition, (9) lift a basket from the seat of a chair to the surface of an adjacent desk, and one additional task not appearing in the Wolf Test (10) flexion and extension at the elbow with the arm in adduction. Tasks 1-7 were executed while the subject was seated, tasks 8-10 were carried out while the subject was standing. All movements were performed with the dominant arm, which was the right arm in all cases. Subjects were told to watch the arm of the metronome to help in timing task movements. Each movement was described and demonstrated; the subjects then performed the task 10 times. After 5 tasks, subjects were given a brief rest.
After completion of the standardized tasks, the recording device was set in ambulatory (offline) mode and subjects performed activities of daily living (ADL) for 5 hours. There were no instructions concerning kind of activities to be engaged in since in this part of the experiment, the objective was to correlate accelerometer and EMG records during unselected, real world activities. After the subject returned to the laboratory, he was asked what kind of activities he had engaged in and these were recorded. Activities included shopping, bicycle repairing, writing, going for a walk, doing the housework, going to a restaurant.

\section{Data Reduction and Data Analysis}

Since acceleration as well as deceleration of a limb may be associated with muscle activity, only the absolute value of accelerometer data was used for analysis. Data analyses were performed by means of the MATLAB ${ }^{\mathrm{TM}}$ computer environment which was installed on a personal computer with a pentium $100 \mathrm{MHz}$ processor. The relation of time series obtained by different sensors and at different recording sites was evaluated by means of Pearson correlation coefficients. Movements of the skeleton, as recorded by accelerometer counts, lag behind initiation of muscle activation; similarly termination of movements of body parts lag behind a cessation of EMG activity. Accordingly, to achieve a temporal equation of accelerometer and EMG recordings for given movements, a time lag of $.5 \mathrm{~s}$ was introduced between the records. Net wrist accelerometer readings were computed by subtracting hip acceleration counts from the wrist acceleration counts.

Multiple correlation coefficients were employed to evaluate the relation between EMG records from the upper and lower arm and from the wrist accelerometer. Metronome beats were represented by a sine-wave at $0.67 \mathrm{~Hz}(40 \mathrm{bpm})$ in order to obtain correlation coefficients between both EMG and accelerometer records and an index of the actual performed movements. In order to determine single values describing EMG and accelerometer records for certain tasks, time series were averaged and the standard deviation was computed for each subject. Subsequently, means and standard deviations were pooled for the six subjects. Pearson coefficients were then computed across tasks. Means and standard deviations of correlation coefficients across subjects or conditions were obtained using Fisher's $z$-transformation. 


\section{Results}

Correlations among time series of wrist accelerometer counts and EMG recordings of lower and upper arm for standardized movements were low; however, there were substantial differences both between tasks and subjects (see Tables 1 and 2). Simple tasks involving repetition of only one type of movement (e.g., task 2 "hand to box" or task 10 "flexion and extension of arm") were found to have a higher convergence of EMG and accelerometer readings than more complex tasks. There were higher correlations between EMG and accelerometer records for the time series obtained while ADL were performed than for the standardized laboratory tasks. A mean Pearson coefficient of $0.32(P<0.01, \mathrm{SD}=0.07)$ was found for the relation of lower arm EMG and wrist accelerometer across points in time, and there was also a significant relation between biceps EMG and wrist accelerometer counts (mean $r=0.40, P<0.01, \mathrm{SD}=0.14$ ). Thus, correlations showed a lower degree of variability across subjects in the ADL as compared to the standardized tasks. As might be expected intuitively, wrist accelerometer readings are determined by both lower and upper arm muscle activity, which in turn do not necessarily occur simultaneously. Consequently, it might be predicted that the correlation of wrist accelerometer signals to EMG activity might be increased by means of multiple correlations that establish a relation between accelerometer activity and the joint EMG activity of both segments of the arm. The results are confirmatory, indicating that a combination of EMG information from the proximal and distal portions of the arm allows more precise prediction of the accelerometer readings than use of just one of the EMG channels. This is true both for ADL (mean multiple $r=0.53, P<0.01, \mathrm{SD}=0.06)$ and standardized tasks (mean multiple $r=0.34, P<0.01, \mathrm{SD}=0.21$ ).

Strong differences across task types were found in terms of the correlations between the metronome beat, which paced movements in the standardized tasks, and the empirical signals (wrist accelerometer, biceps EMG, lower arm EMG) that were recorded during these tasks.

Table 1 Pearson correlation coefficients between lower arm EMG and wrist accelerometer records across points in time for each subject for each of 10 standardized tasks. Means and standard deviations of correlations across subjects for each task are presented in the last two rows.

\begin{tabular}{lllllllllll}
\hline Subject & 1 & 2 & 3 & 4 & 5 & 6 & 7 & 8 & 9 & 10 \\
\hline 1 & .06 & .28 & .18 & .09 & .13 & .27 & .06 & .11 & .19 & .20 \\
2 & .43 & .66 & .16 & .24 & .09 & .30 & .06 & .13 & .01 & .64 \\
3 & .04 & .23 & .40 & .27 & .13 & .20 & .09 & .16 & .07 & .14 \\
4 & .28 & .49 & .16 & .32 & .11 & .29 & .14 & .13 & .14 & .15 \\
5 & .08 & .07 & .26 & .42 & .22 & .39 & .29 & .13 & .24 & .26 \\
6 & .16 & .23 & .35 & .19 & .08 & .01 & .05 & .00 & .22 & .39 \\
\hline Mean & .18 & .34 & .25 & .26 & .13 & .25 & .12 & .11 & .15 & .31 \\
SD & .16 & .25 & .11 & .12 & .05 & .14 & .09 & .06 & .09 & .23 \\
\hline
\end{tabular}

Note: When $r>0.18, P<0.05$; when $r>0.25, P<0.01$

Table 2 Pearson correlation coefficients between biceps EMG and wrist accelerometer records across points in time for each subject for each of 10 standardized tasks. Means and standard deviations of correlations across subjects for each task are presented in the last two rows.

\begin{tabular}{lllllllllll}
\hline Subject & 1 & 2 & 3 & 4 & 5 & 6 & 7 & 8 & 9 & 10 \\
\hline 1 & .25 & .59 & .66 & .21 & .15 & .19 & .14 & .35 & .48 & .22 \\
2 & .28 & .69 & .33 & .15 & .44 & .07 & .22 & .25 & .16 & .59 \\
3 & .53 & .36 & .28 & .16 & .31 & -.11 & .15 & .17 & .18 & .65 \\
4 & .13 & .19 & .13 & .31 & .27 & .06 & .16 & .07 & .08 & .26 \\
5 & .13 & .38 & .33 & .27 & .18 & .25 & .19 & .15 & .26 & .22 \\
6 & -.04 & .19 & .32 & .36 & .22 & .24 & .17 & .24 & .02 & .30 \\
\hline Mean & .22 & .42 & .36 & .24 & .26 & .12 & .17 & .21 & .20 & .39 \\
SD & .21 & .26 & .22 & .09 & .12 & .14 & .03 & .10 & .18 & .24 \\
\hline
\end{tabular}

Note: When $r>0.18, P<0.05$; when $r>0.25, P<0.01$ 
Table 3 Intercorrelations of measures across tasks (1-10) for pooled means (below diagonal) and pooled standard deviations (above diagonal); $n=6$.

\begin{tabular}{|c|c|c|c|c|c|}
\hline & & $\begin{array}{l}\text { Accele } \\
\text { hip }\end{array}$ & $\begin{array}{l}\text { rometer } \\
\text { wrist }\end{array}$ & $\begin{array}{l}\text { EMG } \\
\text { biceps }\end{array}$ & lower arm \\
\hline \multirow[t]{2}{*}{ Accelerometer } & hip & - & -.26 & -.03 & .46 \\
\hline & wrist & -.15 & - & .46 & .37 \\
\hline \multirow[t]{2}{*}{ EMG } & biceps & -.13 & .14 & - & .63 \\
\hline & lower arm & .15 & .65 & .71 & - \\
\hline
\end{tabular}

Note: When $r>0.63, P<0.05$; when $r>0.77, P<0.01$

Measures did not correspond strongly with the pacing metronome beat in complex tasks, but there was a strong relation between metronome beat and signals in tasks 2 (mean $r=0.51$ for the wrist acclerometer, mean $r=0.42$ for the biceps EMG) and 10 (mean $r=0.62$ for the wrist acclerometer, mean $r=0.72$ for the biceps EMG) which, as noted, are simple in character. In general, wrist accelerometer and upper arm EMG records showed a relatively low mean covariation with the metronome pacing of $r$ $=0.31$ and 0.34, respectively, while lower arm EMG seemed to be a less precise predictor of metronome beat (mean $r=0.24)$.

The correlations among pooled means and standard deviations of the signals across tasks are presented in Table 3. They indicate that the means of wrist accelerometer recordings and lower arm EMG covary significantly across tasks $(r=0.65, P<0.05)$, while the relation of means of wrist accelerometer records and biceps EMG $(r=0.14$, n.s. $)$ is negligible. In a similar study, Fahrenberg and coworkers (1996) found a correlation of 0.84 for pooled means between uniaxial accelerosensors and EMG both recorded from the lower leg across 7 tasks. Correlation coefficients for standard deviations of lower arm EMG and wrist accelerometer $(r=0.37)$ as well as for biceps EMG and wrist accelerometer $(r=0.46)$ were not significant.

The computation of net wrist accelerometer readings by subtracting hip acceleration from the wrist accelerogram resulted in correlations between EMGs and net accelerometer readings of approximately zero. Observation of the subjects made the reason for this evident: counts from the hip accelerometer reflected primarily torsional and "jiggling" movements; ambulatory movements contributed only a relatively small number of counts to the record. Thus, the net wrist accelerometer readings are not reported here.

\section{Discussion}

In the present method study we found that when using an individual time series approach, the correlation between
EMG and accelerometer measures was not strong. The correlation between accelerometer counts and EMG activity was in the range $0.3-0.4$ for activities of daily living. Pearson coefficients between shorter time series obtained in the individual laboratory task condition were lower still, being 0.3 at best. This discrepancy between standardized tasks and ADL was mainly due to ADL data segments recorded while the subjects were resting and consequently both measures decreased. When a statistical approach involving the use of pooled means and SDs across subjects was employed, the correlation rose to .65 , but this approach is of less use for the determination of pre- to posttreatment effects for physical rehabilitation interventions than is the analysis of individual time series.

Multiple correlations revealed that both lower and upper arm EMG records were individually related to accelerometer readings, but the EMG records from the two portions of the arm were not significantly related to one another. The consequence was that the use of multiple correlations involving both portions of the arm resulted in better prediction of accelerometer counts than the use of either EMG measure by itself. In general, the data suggest that EMG and accelerometer records index different parameters of movement.

It was found that the relationship between EMG and accelerometer records within a given movement was not linear. In the initiation phase of movement, both measures increase. However, there is an inverse relation between the two measures during the deceleration of movement; the accelerometer gives increased counts but the EMG decreases, reflecting decreased muscle contraction. This disparity was addressed by introducing a time lag between EMG and accelerometer time series. However, this increased the correlation between the two measures only modestly. This is another indication that EMG and accelerometer recordings relate to different aspects of movement. In consequence, the time lag approach to compensate for the delay in accelerometer onset and offset as compared to EMG activity does not at present appear to be useful. In addition, the attempt to partial out acceleration of the arms due to their passive transport during ambulation was not successful. As noted, accelerometer counts from the hip transducer were more influenced by local movements from the center of the body than by locomotor translation.

The present findings have relevance for the assessment of motor activity. Neither the EMG nor the accelerometer alone can be considered to give a complete picture of a person's movements. Consequently, it may be of value to use both measures simultaneously in assessing the amount and nature of movement that subjects are carrying out. This consideration should be of value in designing a measure of the amount of use of an extremity in a rehabilitation setting. 


\section{Acknowledgment}

This research was supported by grants from the Deutsche Forschungsgemeinschaft, the James S. McDowell Foundation, and Grant B95-975R from the Rehabilitation Research and Development Service, U.S. Department of Veterans Affairs.

\section{References}

Andrews, K., \& Stewart, J. (1979). Stroke recovery: He can but does he? Rheumatology and Rehabilitation, 18, 43-47.

Bach, T. M., Reddihough, D. S., Burgess, G., \& Johnson, L. M. (1994). Comparison of subjective and objective measures of movement performance in children with cerebral palsy. Developmental Medicine and Child Neurology, 36, 974-979.

Bouten, C. V., Verboeket van de Venne, W.P., Westerterp, K. R., Verduin, M., \& Janssen, J. D. (1996). Daily physical activity assessment: comparison between movement registration and doubly labeled water. Journal of Applied Physiology, 81, 10191026.

Boyce, W., Gowland, C., Rosenbaum, P., Lane, M., Plews, N., Goldsmith, C., Russell, D., Wright, V., Zdrobov, S., \& Harding, D. (1992). Gross motor performance measure for children with cerebral palsy: Study design and preliminary findings. Canadian Journal of Public Health, 83, Suppl. 2, 34-40.

Comby, B., Chevalier, G., \& Bouchoucha, M. (1992). Anew method for the measurement of tremor at rest. Archives Internationales de Physiologie, de Biochimie et de Biophysique, 100, 73 78.

Duncan, P. W. (1997). Synthesis of intervention trials to improve motor recovery following stroke. Topics in Stroke Rehabilitation, 3, 1-20.

Fahrenberg, J., Förster, F., \& Müller, W. (1996). Laboratory and field studies for improvement of ambulatory monitoring methodology. In J. Fahrenberg \& M. Myrtek (Eds.), Ambulatory assessment (pp. 237-255). Seattle: Hogrefe \& Huber.

Fahrenberg, J., Förster, F., Smeja, M., \& Müller, W. (1997). Assessment of posture and motion by multichannel piezoresistive accelerometer recordings. Psychophysiology, 34, 607-612.

Formisano, R., Pratesi, L., Modarelli, F. T., Bonifati, V., \& Meco, G. (1992) Rehabilitation and Parkinson's disease. Scandinavian Journal of Rehabilitation Medicine, 24, 157-160.

Janz, K. F. (1994). Validation of the CSA accelerometer for assessing children's physical activity. Medicine and Science in Sports and Exercise, 26, 369-375.

Meijer, G. A., Westerterp, K. R., van Hulsel, A. M., \& ten Hoor, F. (1992). Physical activity and energy expenditure in lean and obese adult human subjects. European Journal of Applied Physiology, 65, 525-528.

Otsuka, K., Cornelissen, G., \& Halberg F. (1994). Broad scope of a newly developed actometer in chronobiology, particularly chronocardiology. Chronobiologia, 21, 251-264.

Redmond, D.P., \& Hegge, F.W. (1985). Observations on the design and specification of a wrist-worn human activity monitoring system. Behavior Research Methods, 17, 659-669.
Repp, A. C., Karsh, K. G., Deitz, D. E., \& Singh, N. N. (1992). A study of the homeostatic level of stereotypy and other motor movements of persons with mental handicaps. Journal of Intellectual Disability Research, 36, 61-75.

Richardson, M. T., Leon A. S., Jacobs D. R., Ainsworth, B. E., \& Serfass, R. (1995). Ability of the Caltrac accelerometer to assess daily physical activity levels. Journal of Cardiopulmonary Rehabilitation, 15, 107-113.

Rutter, S. (1994). Comparison of energy expenditure in normalweight and overweight women using the Caltrac Personal Activity Computer. International Journal of Eating Disorders, 15, $37-42$.

Salminen, J. J., Oksanen A., Maki, P., Pentti, J., \& Kujala, U. M. (1993). Leisure time physical activity in the young. Correlation with low-back pain, spinal mobility and trunk muscle strength in 15-year-old school children. International Journal of Sports Medicine, 14, 406-410.

Schandry, R. (1981). Psychophysiologie. Munich: Urban und Schwarzenberg.

Taub, E. (1980). Somatosensory deafferentation research with monkeys: implications for rehabilitation medicine. In L.P. Ince (Ed.), Behavioral psychology in rehabilitation medicine: Clinical applications (pp. 371-401). New York: Williams and Wilkins.

Taub, E., Miller, N.E., Novack, T.A., Cook, E.W. III, Fleming, W.D., Nepomuceno, C.S., Connell, J.S., \& Crago, J.E. (1993). Technique to improve chronic motor deficit after stroke. Archives of Physical Medicine and Rehabilitation, 74, 539-594.

Taub, E., Pidikiti, R.D., DeLuca, S.C., \& Crago, J.E. (1996). Effects of motor restriction of an unimpaired upper extremity and training on improving functional tasks and altering brain/behaviors. In G. Toole \& D. Goode (Eds.), Imaging in neurologic rehabilitation (pp. 133-154). New York City: Demos Vermande.

Taub, E., \& Wolf, S. L. (1997). Constraint induction techniques to facilitate upper extremity use in stroke patients. Topics in Stroke Rehabilitation, 3, 38-61.

Tobler, I. (1993). Aktometrie zur Objektivierung von Schlafstörungen. Therapeutische Umschau, 50, 684-687

Uswatte, G., \& Taub, E. (in press). Constrained-Induced Movement Therapy: new approaches to outcome measurement in rehabilitation. In B.T. Stuss, G. Winocur, \& I.H.Robertson (Eds.), Cognitive neurorehabilitation: A comprehensive approach. New York: Cambridge University Press.

Wolf, S. L., Lecraw, D. E., Barton, L. A., \& Jann, B. B. (1989). Forced use of hemiplegic upper extremities to reverse the effect of learned nonuse among chronic stroke and head-injured patients. Experimental Neurology, 104, 104-132.

Andreas Keil

University of Konstanz

Fachgruppe Psychologie

D-78434 Konstanz, Germany

Tel: +49 7531884003

E-mail: Andreas.Keil@uni-konstanz.de 